J. Bangladesh Acad. Sci., Vol. 42, No. 1, 201-205, 2018

DOI: https://doi.org/10.3329/jbas.v42i2.40053

\title{
FUZZY NORMAL TOPOLOGICAL SPACE IN QUASI-COINCIDENCE SENSE
}

\author{
SAIKH SHAHJ AHAN MIAH ${ }^{1 *}$, RUHUL AMIN ${ }^{2}$ AND SOHEL RANA ${ }^{2}$ \\ Department of Civil Engineering, Pundra University of Science \& Technology, Bogura-5800, \\ Bangladesh.
}

\section{ABSTRACT}

Three notions of normal property in fuzzy topological spaces using quasi-coincidence sense are introduced in this paper and relationship among these and other such notions are established. It is also shown that all these notions satisfy 'good extension' property. It is observed that the notions are preserved under one-one, onto, fuzzy open, fuzzy closed and fuzzy continuous mappings.

Keywords: Fuzzy Topological Space, Fuzzy Normal Topological Space, Mappings, Quasicoincidence.

\section{INTRODUCTION}

In 1965 the concept of fuzzy set was given by L A Zadeh (1965). By using this concept, Chang (1968) defined fuzzy topological spaces. Since then extensive work on fuzzy topological spaces has been carried out by many researchers like Gouguen (1973), Wong (1974), Lowen (1976), Warren (1974), Hutton (1975) and others. Separation axioms are important in fuzzy topological spaces. Many works like D. M. Ali (1990), F. Ali Ahmd (1989), S. S. Miah and M. R. Amin (2017), S. S. Miah, M. R. Amin and M. Jahan (2017), S. S. Miah, M. R. Amin and H. Rashid (2017) on separation axioms have been done by fuzzy mathematicians. Among those axioms, fuzzy normal type is one and it has been already introduced in fuzzy topology. There are many articles on fuzzy normal topological space which are stated by many authors like P. Wuyts and R. Lowen (1983), D. M. Ali (1990), A. C. Guler and Goknur Kale (2015) and other researchers.

The objective of this paper is to further contribute to the development of separation axioms in normal fuzzy topological spaces. In the present paper, fuzzy normal topological space is defined by using quasi-coincidence sense and implications among the present one and other such notions are shown. It is shown that the 'good extension' and order preserving properties hold for the new concepts.

\section{BASIC NOTIONS AND PRELIMINARY RESULTS}

In this section, some concepts which are presented here which will be needed in the sequel. In this paper, $X$ and $Y$ are always denoted as non empty sets and $I=[0,1]$. The class of all fuzzy sets on a non empty set $X$ is denoted by $I^{X}$ and fuzzy sets on $X$ are denoted by $u, v, w$ etc.

Definition 2.1 A function $u$ from $X$ into the unit interval $I$ is called a fuzzy set in $X$. For every $x \in X, u(x) \in I$ is called the grade of membership of $x$ in $u$ (Zadeh 1965).

Definition 2.2 A fuzzy set $u$ in $X$ is called a fuzzy singleton if and only if

\footnotetext{
* Corresponding author: <skhshahjahan@gmail.com>.

${ }^{2}$ Department of Mathematics, Begum Rokeya University Rangpur-5404, Bangladesh.
} 
$u(x)=r, 0<r \leq 1$, for a certain $x \in X$ and $u(y)=0$ for all points $y$ of $X$ except $^{X}$. The fuzzy singleton is denoted by $x_{r}$ and ${ }^{X}$ is its support. The class of all fuzzy singletons in $X$ will be denoted by $S(X)$. If $u \in I^{X}$ and $x_{r} \in S(X)$, then $x_{r} \in u$ if and only if $r \leq u(x)$ (P. Ming and Y. Ming 1980).

Definition 2.3 A fuzzy singleton $X_{Y}$ is said to be quasi-coincidence with $u$, denoted by $x_{r} \bar{q} u$ if and only if $u(x)+r>1$. If $x_{r}$ is not quasi-coincidence with $u$, it is $x_{r} q u$ and defined as $u(x)+r \leq 1$ (Kandil and El-Shafee 1991).

Definition 2.4 Let $f$ be a mapping from a set $X$ into a set $Y$ and $u$ be a fuzzy subset of $X$. Then $f$ and $u$ induce a fuzzy subset $v$ of $Y$ defined by $v(y)=\sup \{u(x)\}$

if $x \in f^{-1}[\{y\}] \neq \varphi, x \in X=0$ otherwise (Chang 1968).

Definition 2.5 Let $f$ be a mapping from a set $X$ into a set $Y$ and $v$ be a fuzzy subset of $Y$. Then the inverse of $v$ written as $f^{-1}(v)$ is a fuzzy subset of $X$ defined by $f^{-1}(v)(x)=v(f(x))$, for $x \in X$ (Chang 1968).

Definition 2.6 Let $I=[0,1], X$ be a non empty set and $I^{X}$ be the collection of all mappings from $X$ into $I$, i.e. the class of all fuzzy sets in $X$. A fuzzy topology on $X$ is defined as a family $t$ of members of $I^{X}$, satisfying the following conditions.

(i) $1,0 \in t$,

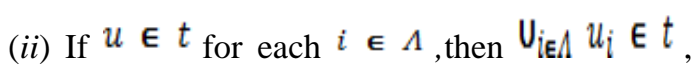

where $\Lambda$ is an index set. (iii) If $w^{v \in t}$ then $u \cap v \in t$ (Chang 1968). The pair $(X, t)$ is called a fuzzy topological space (in short fts) and members of $t$ are called $t$ - open fuzzy sets. A fuzzy set $v$ is called a $t$-closed fuzzy set if $1-v \in t$.

Definition $\quad 2.7 \quad$ The function $f:(X, t) \rightarrow(Y, s)$ is called fuzzy continuous if and only if for every $v \in s_{y} f^{-\mathbf{1}}(v) \in t$ the function $f$ is called fuzzy homeomorphic if and only if $f$ is bijective and both $f$ and $f^{-1}$ are fuzzy continuous (P. Ming and Y. Ming 1980).

Definition 2.8 The function $f:(X, t) \rightarrow(Y, s)$ is called fuzzy open if and only if for every open fuzzy set $u$ in $(X, t), f(u)$ is open fuzzy set in $(Y, s)$ (Malghan and Benchalli 1984).

Definition 2.9 The function $f:(X, t) \rightarrow(Y, s)$ is called fuzzy closed if and only if for every closed fuzzy set $u$ in $(X, t), f(u)$ is closed fuzzy set in $(Y, s)$ (P. Ming and Y. Ming 1980).

Theorem 2.1 A bijective mapping from an $\mathrm{fts}$ $(X, t)$ to an fts $(Y, s)$ preserves the value of a fuzzy singleton (fuzzy point).

Note: Preimage of any fuzzy singleton (fuzzy point) under bijective mapping preserves its value (Amin et al. 2014).

\section{FUZZY NORMAL TOPOLOGICAL SPACE}

In this section, notions and findings are discussed. Some well-known properties are discussed here by using concepts of this paper.

Definition 3.1 A fuzzy topological space $(X, t)$ is called

(a) $F N(i)$ iff $W_{1} \bar{q} W_{2}$ where $W_{1}, W_{2}$ are $t$-closed fuzzy set, there exist $u, v \in N\left(w_{z}, t\right)$ such that ฯ $\bar{q}$ v (D. M. Ali 1990). 
(b) $F N(i i)$ iff $w_{1} \bar{q} w_{2}$ where $w_{1}, w_{2}$ are $t$-closed fuzzy set, there exist $u, v \in N\left(w_{2}, t\right)$ such that $u \cap v=0$.

(c) $F N(i i i)$ iff $w_{1} \cap w_{2}=0$ where $w_{1}$, $W_{\mathbf{2}}$ are ${ }^{t}$-closed fuzzy set, there exist $u, v \in t$ such that $w_{1} \subseteq u, w_{2} \subseteq v$ and $u \bar{q} v$.

(d) $F N(v)$ iff $w_{1} \cap w_{2}=0$ where $w_{1}, w_{2}$ are $t$. closed fuzzy set, there exist $u, v \in t$ such that $w_{1} \subseteq u, w_{2} \subseteq v$ and $u \cap v=0$.

Theorem 3.2 Let $(X, t)$ be a fuzzy topological space. Then the implications $(\mathrm{b}) \Rightarrow(\mathrm{a}),(\mathrm{d}) \Rightarrow(\mathrm{c})$ are true in general.

Proof: $\quad(b) \Rightarrow(a), \quad(d)^{\Rightarrow(c)}$ are obvious since $u \cap v=0$ implies that $u \bar{q} v$.

Now, we shall give a counter example which shows that (a) does not imply (b).

Example 3.3 Let $X=\{x, y\}$ and ${ }^{t}$ be a fuzzy topologyo $\mathrm{n}^{X}$ generated by $\{u, v\} \cup\{$ constants $\}$, where $u(x)=0.4, u(y)=0.2$ and $v(x)=0.3, \quad v(y)=0.6$. Then it is seen that $\left(\mathbb{X}_{,} \quad t\right)$ is $F N(i)$ but not $F N(i i)$. Hence $(a) \Rightarrow(b)$.

The following counter example shows that (c) does not imply (d).

Example 3.4 Let $X=\{x, y\}$ and ${ }^{t}$ be a fuzzy topology on $X$ generated by $\left\{u_{1} \quad v\right\} \cup\{$ constants $\}$, where $u(x)=0.4, u(y)=0.2$ and $v(x)=1, \quad v(y)=0.3$. Then it is seen that $(X, \quad t)$ is $F N(i i i)$ but not FN $(i v)$. Hence $(\mathrm{c}) \nRightarrow(\mathrm{d})$.

Theorem 3.5 The topological space $(X, \quad T)$ is normal iff $(X, w(T))$ is $F N(j)$, where $j=i, i i, i i i, i v$.
Proof: the theorem for $j=i v$ needs to be proved. Proof of others is similar. Let $(X, w(T))$ is FN(iv). It has to be shown that $(X, T)$ is normal topological space. Let $W_{1}, W_{2}$ be $T$ closed sets with $W_{1} \cap W_{2}=\emptyset$. Then it is clear that $\mathbf{1}_{W_{1}}$ and $\mathbf{1}_{W_{2}}$ are fuzzy sets in $w(T)$ and $1_{W_{1} \cap W_{2}}=0$. But $1_{W_{1} \cap W_{2}}=0$ implies $1_{W_{1}} \cap 1_{W_{2}}=0$. Since $(X, w(T))$ is $F N(i v)$, then there exist $u, v \in W(T)$ such that $1_{W_{1}} \subseteq v, 1_{W_{2}} \subseteq u$ and $u \cap v=0$.

Since $u, v \in w(T)$, then $u^{-1}(0,1], v^{-1}(0,1] \in T$. Again since $\mathbf{1}_{W_{1}} \subseteq v$, then $v^{-1}(0,1] \supseteq\left(1_{W_{1}}\right)^{-1}(0,1]=W_{1}$. Similarly, $W_{2} \subseteq u^{-1}(0,1]$.

considering that $u^{-1}(0,1] \cap v^{-1}(0,1] \neq \phi$, then there exists $x \in X$ such that $u(x)>0$ and $v(x)>0$. So $(u \mathbf{n} v)(x)>\mathbf{0}$ which contradicts the fact $u \mathbf{n} v=\mathbf{0}$. Hence the topological space $(X, T)$ is normal.

Conversely, let if topological space $\left(\begin{array}{ll}( & T) \\ & \text { be }\end{array}\right.$ normal. It has to be shown that $(X, w(T))$ is FN(iv). Let $W_{1}, W_{2}$ be $w(T)$-closed with $W_{1} \cap W_{2}=0$. Then, $w_{1}{ }^{C}, W_{2}{ }^{C} \in W(T)$ and $\left(w_{1}^{C}\right)^{-1}(0,1],\left(w_{2}^{C}\right)^{-1}(0,1] \in T$.

Now,

$\left(\left(w_{1}\right)^{-1}(0,1]\right)^{c}=w_{1}^{-1}\{1\}$ and $\left(\left(w_{2}^{c}\right)^{-1}(0,1]\right)^{c}=w_{2}^{-1}\{1\}$. It is clear that $w_{1}^{-1}\{1\}$ and $w_{z}^{-1}\{1\}$ are closed sets in $T$. Since $(X, T)$ is normal, then there exist $U, V \in T$ Such that

\section{${W_{1}}^{-1}\{1\} \subseteq V, W_{2}^{-1}\{1\} \subseteq U$ and $U \cap V=\emptyset$.}

Now, it is clear that $\mathbf{1}_{V}, \mathbf{1}_{U} \in w(T)$ and $\mathbf{1}_{U} \cap 1_{V}=\mathbf{0}$ as $\mathbf{1}_{U n V}=\mathbf{0}$ implies $\mathbf{1}_{U} \cap \mathbf{1}_{V}=\mathbf{0}$.

Hence, ${ }_{w_{1}^{-1}[1]}=w_{1} \subseteq 1_{V}, 1_{w_{2}}^{-1}[1]$

$=w_{\mathbf{2}} \subseteq 1_{U}$ and $1_{U} \cap 1_{V}=0$. 
Therefore, $(X, w(T))$ is $F N(i v)$. The proof is complete.

The following theorem states the hereditary property of the given concepts.

Theorem 3.6 Let $(X, t)$ be a fuzzy topological space, $A \subseteq X$, and

$t_{A}=\left\{\frac{u}{A}: u \in t\right\}$, then $(X, t)$ is $F N(j) \Rightarrow\left(A, t_{A}\right)$ is $F N(j)$ for $j=i, i i, i i l, i v$.

Proof: The proof is obvious.

Theorem 3.7 Let $\left(X_{,} \quad t\right)$ and $\left(Y, t_{1}\right)$ be two fuzzy topological spaces and $f: X \rightarrow Y$ be bijective, fuzzy continuous and fuzzy open mapping. Then

$(X, t)$ is $F N(j) \Rightarrow\left(Y, t_{1}\right)_{\text {is }} F N(j)$, where $j=i, i i, i i i, i v$.

Proof: Suppose the fuzzy topological space $(X, t)$ is $F N(i i i)$. It has to be shown that $\left(Y, t_{1}\right)$ is $F N(i i i)$. Let $w_{1}, w_{2}$ be $t_{1 \text {-closed }}$ with $w_{1} \cap w_{2}=0$. Then $f^{-1}\left(w_{1}\right)$, $f^{-1}\left(w_{2}\right) \in t^{C}$ as $f$ is fuzzy continuous . Let $x \in X$ then, we have $f(x)=y$, for $\mathrm{y}$ in $\mathrm{Y}$. Now, for all $x \in X$, it is $\left(f^{-1}\left(w_{1}\right) \cap f^{-1}\left(w_{2}\right)\right)(x)$

$=w_{1}(f(x)) \cap w_{\mathbf{2}}(f(x))$

$=w_{1}(y) \cap w_{2}(y)=0$, as $w_{1} \cap w_{2}=0$

Since $(X, t)$ is $F N(i i i)$, there exist $u, v \in t$ such that $f^{-1}\left(w_{1}\right) \subseteq u, f^{-1}\left(w_{2}\right) \subseteq v$ and $u \bar{q} v$.

Now, $f^{-1}\left(w_{1}\right) \subseteq u, f^{-1}\left(w_{2}\right) \subseteq v$ imply $w_{2} \subseteq f(v), w_{2} \subseteq f(v)$ as $f$ is bijective.

Also $u q v$ implies that $\mathrm{u}(x)+v(x) \leq 1$ for all $x \in X$.
Now, for all $f(x) \in Y$, we have $f(u) f(x)+f(v)(f(x))$ $=u(x)+v(x) \leq 1$ as $f$ is bijective. Thus, $f(u) \bar{q} f(v)$. Now, it is clear that $f(u), \quad f(v) \in t_{1}$ as $f$ is fuzzy open. So, $f(u), f(v) \in t_{1}$ such that $w_{2} \subseteq f(v)$,

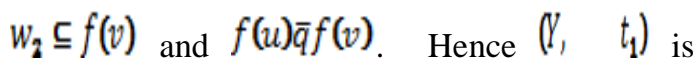
FN(iii). Similarly, one can prove the theorem for $j=i, i i, i v$.

Theorem 3.8 Let $(X, t)$ and $\left(Y, t_{1}\right)$ be two fuzzy topological spaces and $f: X \rightarrow Y$ be bijective, fuzzy continuous and fuzzy closed function. Then

$\left(Y, t_{1}\right)$ is $F N(j) \Rightarrow(X, t)$ is $F N(j)$,

where $j=i, i i, i i i_{,} i v$.

Proof: Let the fuzzy topological space $\left(Y, t_{1}\right)$ be FN(iii). It has to be shown that $(X, t)$ is FN(iii). Let $w_{1}, w_{2}$ be t-closed with $w_{1} \cap w_{2}=0$. Then $f\left(w_{1}\right), f\left(w_{2}\right) \in t_{1}{ }^{C}$ as $f$ is fuzzy closed. Now, for all $y \in Y$, it is

\section{$\left(f\left(w_{1}\right) \cap f\left(w_{2}\right)\right)(y)$}

$=\left(f\left(w_{1}\right) \cap f\left(w_{2}\right)\right)(f(x))$

$=f\left(w_{1}\right)(f(x)) \cap f\left(w_{2}\right)(f(x))$

$=w_{1}(x) \cap w_{2}(x)=0$, since $y=f(x)$ and $f$ is bijective.

Since $\left(Y, t_{1}\right)_{\text {is }} F N(i i i)$, then there exist $u, v \in t_{1}$ such that

$f\left(w_{1}\right) \subseteq u, f\left(w_{2}\right) \subseteq v$ and $u \bar{q} v$.

Now, it is seen that 


$$
\begin{aligned}
& f\left(w_{1}\right) \subseteq u, f\left(w_{2}\right) \subseteq v \text { imply that } w_{1} \subseteq f^{-1}(u), \\
& w_{2} \subseteq f^{-1}(v) \text { as } f \text { is bijective Again, }
\end{aligned}
$$

$u q v$ implies that

$$
u(y)+v(y) \leq \mathbf{1} \text {, for all } y \in Y .
$$

Now, for all $x \in X$, it is

$f^{-1}(u)(x)+f^{-1}(v)(x)$

$$
=u(f(x))+v(f(x))=u(y)+v(y) \leq 1
$$

Hence, $f^{-1}(u) \bar{q} f^{-1}(v)$.

Since $f$ is fuzzy continuous, then $f^{-1}(u), \quad f^{-1}(v) \in t$. So, it becomes $f^{-1}(u), \quad f^{-1}(v) \in t$ such that $w_{1} \subseteq f^{-1}(u)$, $w_{2} \subseteq f^{-1}(v)$ and $f^{-1}(u) \bar{q} f^{-1}(v)$. Hence $\left(X_{1}, t\right)$ is $F N(i i i)$. Similarly, it can be proved for $j=i, i i, i v$.

\section{REFERENCES}

Ahmd, Fora Ali. 1989. Separations axioms for fuzzy spaces, Fuzzy Sets and Systems, 33: 59-75.

Ali, D. M. 1990. On certain separation and connectedness concepts in fuzzy topology, $\mathrm{PhD}$, Banaras Hindu University, India.

Amin, M. R., D. M. Ali and M. S. Hossain. 2014. On $\mathrm{T}_{0}$ fuzzy bitopological spaces, Journal of Bangladesh Academy of Sciences, 32(2): 209217

Chang, C. L. 1968. Fuzzy topological spaces, Journal of Mathematical Analysis and Application, 24: 182-192.

Goguen, T. A. 1973. Fuzzy Tychonoff theorem, Journal of Mathematical Analysis and Application, 43: 734-742.

Guler, A. C. and Goknur Kale. 2015. Regularity and normality in soft ideal topological spaces, Anals of Fuzzy Mathematics and Informatics, 9(3): 373-383.

Hossain, M. S. and D. M. Ali. 2007. On $T_{1}$ fuzzy bitopological spaces, Journal of Bangladesh Academy of Sciences, 31: 129-135.

Hutton, B. 1975. Normality in fuzzy topological spaces, Journal of Mathematical Analysis and Application, 50: 74-79.
Kandil and El-Shafee. 1991. Separation axioms for fuzzy bitopological spaces, J. Inst. Math. Comput. Sci. 4(3): 373-383.

Lowen, R. 1976. Fuzzy topological spaces and fuzzy compactness, Journal of Mathematical Analysis and Application, 56: 621-633.

Malghan, S. R. and S. S. Benchalli. 1984. On open maps, closed maps and local compactness in fuzzy topological spaces, Journal of Mathematical Analysis and Application, 99(2): 338-349.

Miah, S. S. and M. R. Amin. 2017. Certain properties on fuzzy $\mathrm{R}_{0}$ topological spaces in quasicoincidence sense, Annals of Pure and Applied Mathematics, 14(1): 125-131

Miah, S. S. and M. R. Amin. 2017. Mappings in fuzzy Hausdorff spaces in quasi-coincidence sense, Journal of Bangladesh Academy of Sciences, 41(1): 47-56.

Miah, S. S., M. R. Amin and H. Rashid. 2017. T 1 type Separation on Fuzzy Topological Spaces in Quasi-coincidence Sense, Journal of Mechanics of Continua and Mathematical Sciences, 12(1): 45-56.

Miah, S. S., M. R. Amin and M. Jahan. 2017 Mappings on fuzzy $\mathrm{T}_{0}$ Topological Spaces in Quasi-coincidence Sense", Journal of Mathematical and Computational Science, 7(5): 883-894.

Ming, Pu. Pao. and Liu Ying Ming. 1980. Fuzzy topology I. neighborhood structure of a fuzzy point and Moore-Smith convergence, Journal of Mathematical Analysis and Application, 76: 571599.

Ming, Pu. Pao. and Liu Ying Ming. 1980. Fuzzy topology II. product and quotient spaces, Journal of Mathematical Analysis and Application, 77: 20-37.

Warren, R. H. 1974. Continuity of mappings in fuzzy topological spaces, Notices A. M. S. 21: A-451.

Wong, C. K. 1974. Fuzzy topology: product and quotient theorem, Journal of Mathematical Analysis and Application, 45: 512-521.

Wuyts, P. and R. Lowen. 1983. On separation axioms in fuzzy topological spaces, fuzzy neighborhood spaces, and fuzzy uniform spaces, Journal of Mathematical Analysis and Application, 93: 27-41.

Zadeh, L. A. 1965. Fuzzy sets, Information and Control, 8: 338-353.

(Received revised manuscript on 16 October 2018) 